\title{
Logical Sensor Network: An Abstraction of Sensor Data Processing over Multidomain Sensor Network
}

\author{
Naoya Namatame, ${ }^{1}$ Jin Nakazawa, ${ }^{2}$ and Hideyuki Tokuda ${ }^{1}$ \\ ${ }^{1}$ Graduate School of Media and Governance, Keio University, Kanagawa 2520882, Japan \\ ${ }^{2}$ Faculty of Environment and Information Studies, Keio University, Kanagawa 2520882, Japan \\ Correspondence should be addressed to Naoya Namatame, namachan@ht.sfc.keio.ac.jp
}

Received 1 October 2012; Accepted 22 October 2012

Academic Editors: J. Li and Y.-C. Wang

Copyright (C) 2012 Naoya Namatame et al. This is an open access article distributed under the Creative Commons Attribution License, which permits unrestricted use, distribution, and reproduction in any medium, provided the original work is properly cited.

\begin{abstract}
This paper focuses on a sensor network virtualization over multidomain sensor network and proposes an abstraction called "logical sensor network (LSN)" for sensor data processing. In the proposed abstraction, processing is a directed acyclic graph that consists of nodes and streams, which represents a small data processor and communication rules between them, respectively. We have added a notion of a trigger to this graph. A trigger represents a timing of the process execution. We have implemented the middleware named LSN-Middle to run a virtualized sensor network and proved its feasibility.
\end{abstract}

\section{Introduction}

In a future where sensor networks are ubiquitously around us, applications should interact with multiple sensor networks that belong to different domains. Many efforts to make a platform that provides applications a transparent access to sensor data from existing sensor networks with different domains have been made [1-8]. We call this type of platforms "multidomain sensor network (MDSN)." Sensor networks in MDSN include various types of hardware, are organized by different institutions, and are distributed over the world. Utilizing MDSN from applications has two undesirable features that are (1) sensor heterogeneity and (2) raw sensor data delivery. This means applications can only receive raw sensor data with heterogeneous data units and they are responsible for the data translation or the data preprocessing phases. This is caused by a feature that programmers cannot neither configure nor program the sensor networks which consist MDSN. Thus, we consider that an application development over MDSN will be more complicated than the development over a single homogeneous sensor network on which an application programmer has a full configurability and programmability.

For these undesirable features of MDSN, a virtualization of a sensor network can be an effective solution. Sensor network virtualization over MDSN can provide a dedicated sensor network to each application as shown in Figure 1. This figure illustrates application and physical, multidomain and virtualized sensor network. MDSN aggregates physical sensor networks that have been installed for specific purposes [9-13]. And virtualized sensor networks recompose MDSN and create sensor networks for arbitrary applications with arbitrary specifications. Many researchers have been investigating middleware which offers applications to create a virtualized sensor network [14-17]. The middleware allows an application to define a virtualized sensor network. The application can be developed and operated as if it has a dedicated sensor network with a preferred specification. Virtualized sensor network offers application programmers to define sensors and sensor data processing. These features cope with the two undesirable features of MDSN we have mentioned, respectively.

To enable application programmers to define a virtualized sensor network, well-defined abstractions for both sensor and sensor data processing are needed. Although the existing middleware researches and standardizations [18] have well discussed the abstraction of sensors, they have not well investigated the abstraction of sensor data processing. In this research, we propose a sophisticated abstraction for sensor data processing which enables programmers to express 


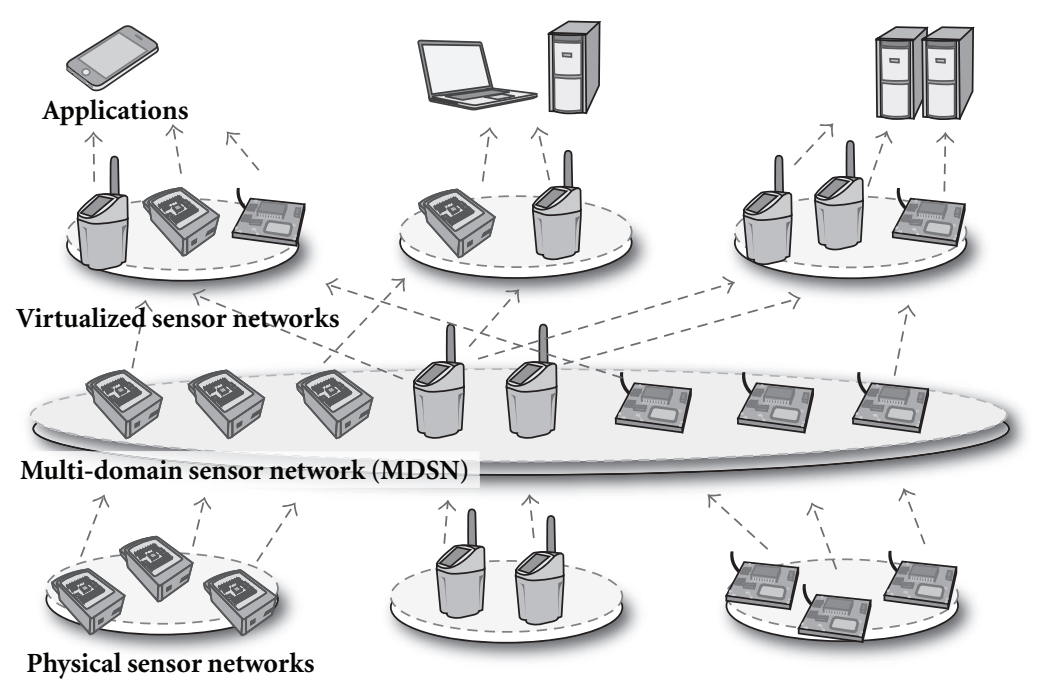

FIGURE 1: Architecture of future sensor network environment.

stateful processing, asynchronous eventing, and multistage data passing. We have also implemented a middleware, which runs virtual sensor networks.

This paper is organized as follows. First, we clarify the features of MDSN and how the sensor network virtualization can contribute to the application development over MDSN in Section 2. Also the problem of the existing researches of the virtualized sensor network will be organized in the section. Section 3, then, proposes LSN and explains details about the abstraction of sensor data processing. Sections 4 and 5 provide implementation and evaluation of LSNMiddle. Section 6 examines related researches. Finally, we summarize this paper in Section 7.

\section{Applications on Multidomain Sensor Network}

MDSN is a platform that provides applications a transparent accessibility to sensor data from existing sensor networks. To describe usages and features of MDSN, we give an example scenario of an application called "Just-In-Time Flurry Forecast."

Just-In-Time Flurry Forecast. On a sunny day, Alice decides to go shopping to a supermarket nearby her house on foot. On the way to the supermarket, her smartphone suddenly alarms her. She picks up her phone and sees a message that says it will be raining so hard in 5 minutes where she is walking now. Of course she do not have an umbrella, she starts to walk a little faster towards her destination. The flurry starts so hard when she just arrives at the supermarket.

2.1. Applications. The notion of "Just-In-Time Flurry Forecast" is derived from an idea that an application provides a microscale weather forecast of where you are right now. The features of MDSN, which offers applications to access sensor data that published by others, are necessary to make this application possible. However, there are two undesirable features to implement this kind of application over MDSN.

(i) Sensor Heterogeneity. Since MDSN consists of physical sensor networks that belong to a large number of institutions and individuals, there is a huge variety in sensors. Therefore, it includes many data units to describe a single physical information. For example, in Just-In-Time Flurry Forecast scenario, the application needs to detect a rain amount $(\mathrm{mm} / \mathrm{h})$ within $5 \mathrm{~km}$ from the application's location. However, MDSN may contain various data units ( $\mathrm{mm} / 10 \mathrm{mins}$, $\mathrm{mm}$ /hour, $\mathrm{mm} /$ day), the application have to translate sensor data with those units into one unit which is $\mathrm{mm} / 10 \mathrm{mins}$ in this case. In addition, the data sources may change when the location of the application moves. Therefore, the application needs to check every sensor data and translate it before the data processing phase.

(ii) Raw Sensor Data Delivery. Before providing data for high-level data processing, which we call an application's main logic, processing steps of raw sensor data into characteristic values are necessary. For example, Just-In-Time Flurry Forecast needs (1) noise filtering each sensor data, (2) averaging multiple sensor data located in certain areas, and (3) calculating a direction of the rain travel, these are the required processing steps. Then, the application's main logic finally calculates how long it takes before flurry arrives at the location of the application and decides whether a notification should be sent or not. Although these steps should be done in a sensor network $[19,20]$, it is impossible to program sensor networks that consist MDSN since they do not belong to the application programmer. Thus, the application needs to receive raw sensor data and is responsible for process it before its main logic.

2.2. Sensor Network Virtualization for MDSN. Sensor network virtualization can be a solution for these features. A 


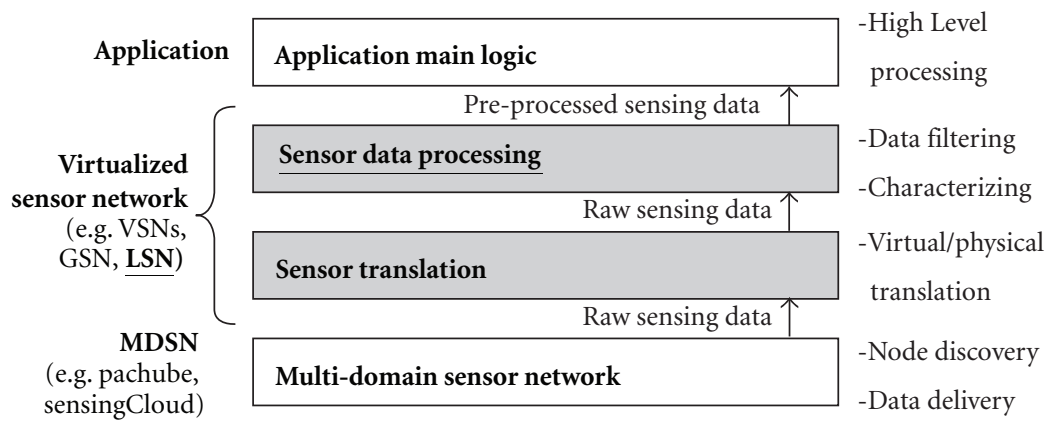

FIGURE 2: Steps required to utilize MDSN from application and LSN's responsibility.

virtualized sensor network that is defined by an application can act as a dedicated sensor network for the application. There are some researches proposing methods to create virtual sensor networks over MDSN [14-16]. They propose virtual sensors that translate different sensor specifications into one to hide heterogeneity of sensors and virtual sensor programs that offer to process sensor data before providing it to applications. The architecture of MDSN, middleware, and application is shown in Figure 2, following researches discussed on both abstractions of sensor and sensor data processing.

Global sensor network (GSN) $[15,16]$ is a research that proposed a mechanism for enabling a concept of virtual sensors and virtual sensor data processing. In the research, the authors have proposed a virtual sensor, which is an abstraction of sensors and sensor data. GSN supports four features to simplify a development of portable application over MDSN, which are sensor data discovery, distributed querying, filtering, and combining multiple sensor data. Virtual sensor in GSN consists with metadata, structures of input/ output data, data processing function, and operational properties. The output values are provided with a certain rate according to a virtual sensor specification.

Virtual sensors [14] are an abstraction of a sensor network and sensor data as well. They focused on providing applications indirect measurement of abstract conditions that need to process multiple sensor data to figure out. Their virtual specification includes a type of input/output data streams, data stream processing functions, and an aggregation frequency. Their virtual sensor abstraction and GSN's abstraction share many features in common.

2.3. Problem. Existing researches of MDSN middleware are not strongly focused on sensor data processing although they have well discussed virtual sensors. Their abstractions of sensor data processing are a single function that has multiple inputs and one output. However, it is insufficient for expressing the sensor data processing for not supporting the following three requirements that we have organized. The application scenario of "Just-In-Time Flurry Forecast" illustrates the three requirements.

(i) Stateful Processing. Some processes may use the former output results or values related to former inputs in their process. These states cannot be stored in a single function that is introduced in the related researches. For example, a noise filter such as lowpass filter that is mentioned in the scenario requires historical sensor data to calculate the result.

(ii) Asynchronous Eventing. Some processes may wait to execute their process until all the available data to be delivered and other processes may want to execute its process every delivery of sensor data. Process execution eventing is not considered in the related researches. For example, a noise filter should be executed on every data delivery, on the other hand, averaging should wait for its execution until a certain amount of sensor data to be delivered.

(iii) Multistage Data Passing. Some processes may need the results of many different processes which are executed asynchronous timing. Multistage data passing cannot be expressed in a synchronous function as introduced in the related researches. For example, the application scenario requires three steps to calculate final output.

The sensor data processing abstraction should be able to express these three aspects. This research will focus on these aspects of sensor data processing and propose an abstraction of sensor data processing that can be used in MDSN middleware.

\section{Logical Sensor Network: Abstraction for Sensor Data Processing}

We propose a simple abstraction of sensor data processing named "Logical Sensor Network (LSN)" that has ability to express stateful data processing, asynchronous eventing, and multistage data passing. This model is a directed graph in which a node (oval) represents a small data processor and a stream (arrow) represents communication rules between nodes. Also, a node can select its process execution timing with a trigger. Top-level nodes and A bottom-level node of a graph, which are exceptions, represent data sources and a data sink, respectively. Data sources are expressed abstractly, so that physical sensors that match to the condition will be placed. With this simple abstraction, sensor data processing in a virtualized sensor network for MDSN becomes simple and expressive. Figure 3 shows a LSN that expresses sensor data processing in the scenario of Just-In-Time Flurry 


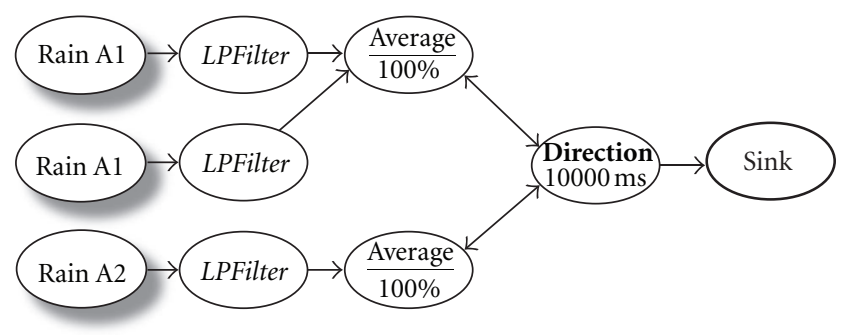

FIGURE 3: A simplified LSN expression of sensor data processing used in Just-In-Time Flurry Forecast scenario.

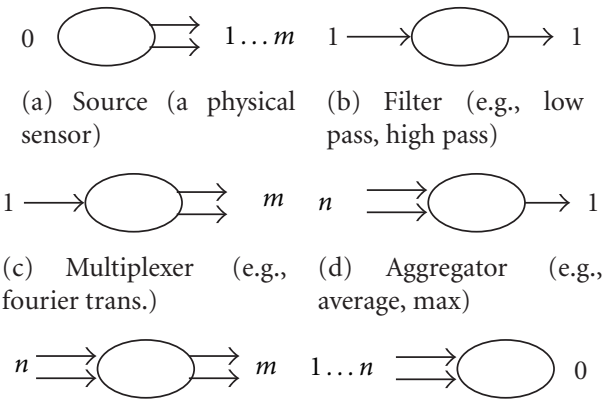

(e) Organizer (e.g., (f) Sink (an application) probability dist.)

FIgURE 4: Comprehensive combination of numbers of input and output stream for a node.

Forecast. Ovals and arrows represent nodes and streams, respectively. Fonts appeared in the nodes represent a type of a trigger.

The graph shows processing steps of data from rain sensors of two areas (Rain A1, Rain A2). Each stream from these nodes are connected to a node that represents processing. Lowpass filtering processes (LPFilter) are connected from nodes of Rain A1 and Rain A2 and execute filtering process when the data arrives from these nodes. These lowpass filtered data are gathered into area averages (Average). Unlike LPFilter, average waits its execution until all the connected streams to send at least a data. At last, a processor that calculates the direction of the rain travel (Direction) executes its process every 10 seconds. This node retrieves the latest value from nodes of Average on its execution. Then it pushes the result value to a sink node. In the following section, we look at each component of the abstraction and explain in details.

3.1. Node. The abstraction of node represents a data process. Node can have $n$ input streams from other nodes and $m$ output streams to other nodes as their input stream. Figure 4 shows possible combinations of the number of input and output streams. Each node contains states that are available as long as the node exists. This feature enables stateful data processing. Source, that is a node with no input stream, is mapped to physical sensor and provides sensor data. Sink, that is a node with 0 output stream, represents an interface to an application. Input streams of a sink can only be accessed by an application. Therefore, source and sink do not contain
TABLE 1: Types of nodes.

\begin{tabular}{lc}
\hline Name & Description \\
\hline Process & Process $n$ inputs and sends $n$ outputs \\
Source & Mapped to physical sensors and provides sensor data \\
Sink & An interface to an applications \\
\hline
\end{tabular}

TABLe 2: Definition of source nodes (Rain A1).

\begin{tabular}{lc}
\hline Key & Value \\
\hline Data kind & Temperature \\
Data unit & Celsius \\
Data type & Number: double \\
Sampling & $3000 \mathrm{~ms}$ \\
Target number & All available \\
Context & Within $5 \mathrm{~km}$ from current loc. \\
\hline
\end{tabular}

processing aspect. All input and output stream combinations which are shown in the Figure 4 can express various kinds of processing that are necessary for sensor data processing, especially preprocessing level. Figures 4(b), 4(c), 4(d), and 4(e) can be categorized as a processor.

They can be categorized into three types as shown in Table 1, which are process, source, and sink.

The number and entity of source nodes need to be changed dynamically since LSN should support the idea of virtual sensors. For this reason, source nodes should be defined in an abstract way. Source node should be defined with a data kind (e.g., temperature), a data unit (e.g., celsius), a data type (e.g., numeric: double), a sampling rate (3000 ms), a total number, and a condition (e.g., target area).

In Figure 3, Rain A1 and Rain A2, that are expressed as a shaded oval, are sources. Sink, that is shown as heavy lined node represents sink, and the other nodes, which are LPFilter, Average, and Direction, represent process. Rain A1 is defined as Table 2. Rain A2 are defined just same as Rain A1 with different context.

3.2. Trigger. Trigger is an element that executes a node's process. Each process node has one trigger. Trigger can be categorized into three types based on an aspect of a timing of a process execution. The categories are shown in Table 3. Flow is a type that executes a process when one of connected input streams brings sensor data. On the other hand, Rendezvous waits the execution until a specified percentage of the connected input streams brings their sensor data. And Timer executes the process periodically with a specified interval. The concept of the trigger enables programmers to easily handle an asynchronous eventing aspect of sensor data streams.

In Figure 3, the node with italic font has a flow type trigger. LPFilter has flow type trigger in this case. Nodes with underlined font, that are Average, have a rendezvous trigger. The percentage written under the node name specifies the requirement. Direction, that is expressed with bold font, is a node with a timer trigger. The time written under the node name represents the execution interval. 
TABLE 3: Types of trigger.

\begin{tabular}{lcc}
\hline Name & Representation & Description \\
\hline Flow & Italic & Each time one of the data from connected input arrives \\
Rendezvous & Underlined & When the data from all connected input arrive \\
Timer & Bold & At a certain time cycle \\
\hline
\end{tabular}

TABle 4: Process execution timing and available data.

\begin{tabular}{lcc}
\hline Type & Timing & Available data \\
\hline \multirow{3}{*}{ Flow } & $d 1$ & $d 1$ \\
& $d 2$ & $d 2$ \\
& $d 3$ & $d 3$ \\
\multirow{2}{*}{ Rendezvous } & $d 4$ & $d 4$ \\
\multirow{2}{*}{ Timer } & $d 4$ & $d 1, d 2, d 3, d 4$ \\
& $t 1$ & $d 1$ \\
& $t 2$ & $d 2, d 3$ \\
\hline
\end{tabular}

TABLE 5: Types of stream.

\begin{tabular}{lcc}
\hline Name & Representation & Description \\
\hline Push & A simple arrow & $\begin{array}{c}\text { Sends data when a process is } \\
\text { executed }\end{array}$ \\
Pull & $\begin{array}{c}\text { A two direction } \\
\text { arrow }\end{array}$ & $\begin{array}{c}\text { Sends data when requested from } \\
\text { the other node }\end{array}$ \\
\hline
\end{tabular}

Figure 5 shows the model of a data arrival to a node from three connected streams. A painted circles $(d 1-d 4)$ on each stream represent sensor data coming into the node. Data with smaller number arrives at the node earlier. The dotted vertical lines $(t 1, t 2)$ represent time that a timer trigger executes the process. When data arrives at the node from these streams, Table 4 shows the timing of the process execution and available sensor data at that time for each trigger type. In this case, when the node adopts a rendezvous trigger, the node receives two data from the same stream, namely, $d 1$ and $d 3$. In such case, whether utilizing the latest data, that is $d 3$, or utilizing average of both data depends on the implementation of the node. LSN abstraction does not concern this issue.

3.3. Stream. Stream connects two nodes and expresses a data communication as shown in Table 5. An output value from a node goes through a stream and provided to the other node as input value. A stream has two types that represent different communication aspects. One is called push that pushes data to the other node after a process execution. A simple arrow represents push link. The other is called pull which retrieves sensor data from the destination node when a node executes its process. An arrow with backward direction tail represents pull link. Connecting nodes by streams enables multistage data passing. And each node only receives data that are relevant to its process.

In Figure 3, data will be pushed by former nodes, as for LPFilter and Average. On the other hand, Direction has pull

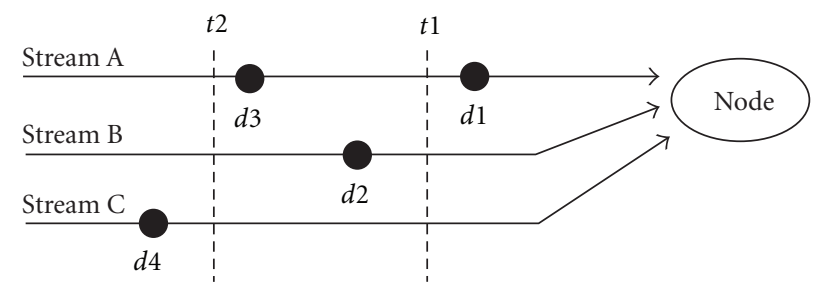

$$
\begin{array}{r}
\text { Data } \\
--- \text { Time }
\end{array}
$$

Figure 5: Data arrival model for a node in LSN.

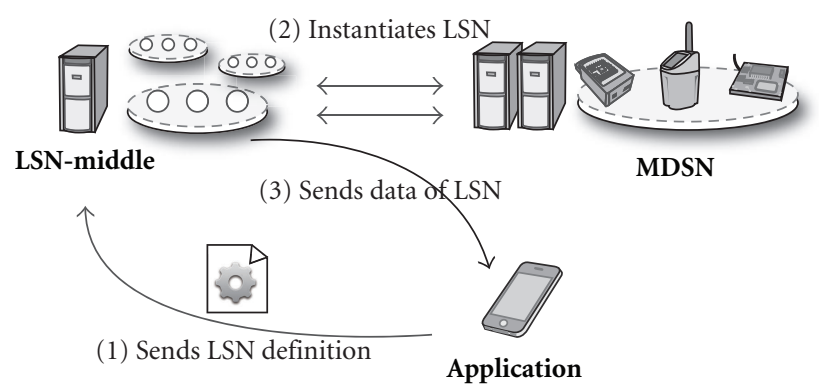

FIGURE 6: The steps for applications to instantiate LSN and receive data.

stream towards Average, which means Direction retrieves the latest average data on its process execution.

\section{Framework Implementation}

We have implemented a framework and middleware for defining and running LSN that is defined according to our abstraction of sensors and sensor data processing. Figure 6 shows how an application instantiate LSN. (1) First, an application sends a definition of LSN to a server with LSNMiddle. (2) When LSN-Middle receives the definition, it instantiate LSN according to the definition. Then it receives and processes sensor data from MDSN.(3) Finally, LSNMiddle sends processed sensor data to the application. Definition of LSN includes source sensor definition (such as Table 2) and a program of LSN that is described in this section. All or a part of a LSN definition can be published to the public and other applications can reuse it.

In this section, we will describe our java framework implementation for programming sensor data processing with the abstraction that we have discussed in the last section using LSN in Figure 3. Each node type shown in Figure 4 has a corresponding java abstract class and an application 


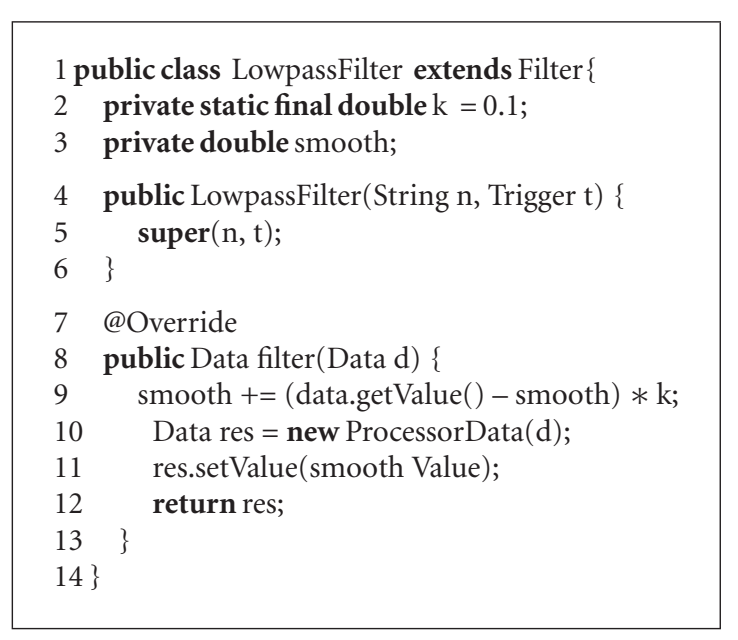

Listing 1: Source code for filter node (lowpass filter) node in Java.

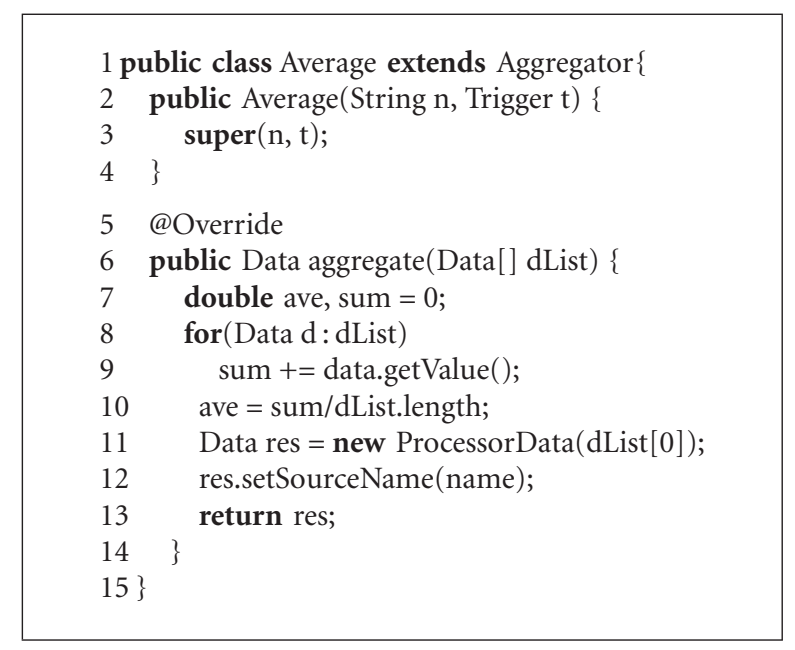

Listing 2: Source code for aggregator node (average) in Java.

developer should extend those abstract classes and override a data processing method to create his/her original processor node. Listing 1 is an example implementation for executing a lowpass filter and Listing 2 is an example source for an averaging process. Processor nodes can be instantiated with a name and a trigger object.

Once an application developer implemented processor nodes, he/she needs to connect them and create LSN. LSN that is shown in Figure 3 can be expressed as the source code shown in Listing 3. LSN can be defined within a java class that extends Logical Sensor Network class. The subclass should implement onInit() and onUpdate() methods. The application developer should write initiation code of LSN in onInit() and reconstruction code in onUpdate() for when binding of logical/physical sensors are changed. In the case of Listing 3, instances of Average class are not newly instantiated, but instances of LowpassFilter class are newly instantiated according to new sources and reconnected to the instances of Average class in onUpdate(). setStream(String,
Node) or addStream(String, Node) is used to connect nodes. These methods need stream name and destination node as parameters. Stream name is useful for distinguishing the stream to read/write data when multiple input/output streams are there.

LSN source code is compiled and sent to LSN-Middle in jar file by an application and executed remotely. When the application sends a connect request with its current context to the LSN, LSN collaborates with MDSN and starts to send sensor data. Listing 4 shows application code that creates, connects, and updates a LSN instance on the remote LSNMiddle.

\section{Evaluation}

We have done an evaluation test to ensure that the LSNMiddle's design and implementation is feasible. In the evaluation, we have compared the time that required for a smartphone to acquire data from MDSN and process it with and without LSN-Middle. The LSN that we have used for the evaluation is shown in Figure 7. This comparison can discover whether LSN-Middle's overhead cost for sending or dynamically loading jar file is acceptable or not. The test requires a smartphone to acquire an average value of $\{10,50,100,500,1000\}$ lowpass filtered sensor data from MDSN with 2 patterns: with and without LSN-Middle. The test is conducted within a private network with a simulated delay and bandwidth. Bandwidth is set to $3000 \mathrm{kbps}$ and RTT between the smartphone (Sensation, $1.2 \mathrm{GHz}$ Dual-Core Qualcomm MSM8260, Android 2.3.3) and LSN-Middle (MacBook Air, $1.8 \mathrm{GHz}$ Intel Core i7, MAC OS X 10.7) or MDSN platform is 25.8 (stddev: 1.44). MDSN platform that we have used is prepared for this evaluation and it generates simulated sensor data. A client that needs data from the MDSN sends a query with sensor conditions, and first the client gets an address list of matched sensors and then sensor data follows. All the specified amount of sensor data is generated within $3000 \mathrm{~ms}$ and sent to the client. The last sensor data is generated exactly on $3000 \mathrm{~ms}$ from the request as an anchor. We have measured a time that the smartphone took to get the average value from the time sending a request. This time includes the required time for MDSN to finish generating all the sensor data. Therefore, we have defined response time that is related to the efficiency to be MeasuredTime - $3000 \mathrm{~ms}$.

The graph in Figure 8 shows the result. The error bar represents standard deviation. As a result, the performance with LSN-Middle is $12.57 \%$ better in average. This means, in spite of the additional required steps, the performance is better with LSN-Middle. There are two reasons that the response time becomes larger as the number of sensor data becomes larger. One is that sensor data processing time becomes larger in proportion to the number of sensor data. The other reason is that the cost of sending a list for sensor nodes from MDSN is becoming larger. The performance with LSN becomes better because these tasks are executed in the LSN-Middle where there are richer computational resources than that of a smartphone. When processing a heavier load task than averaging, the performance difference 


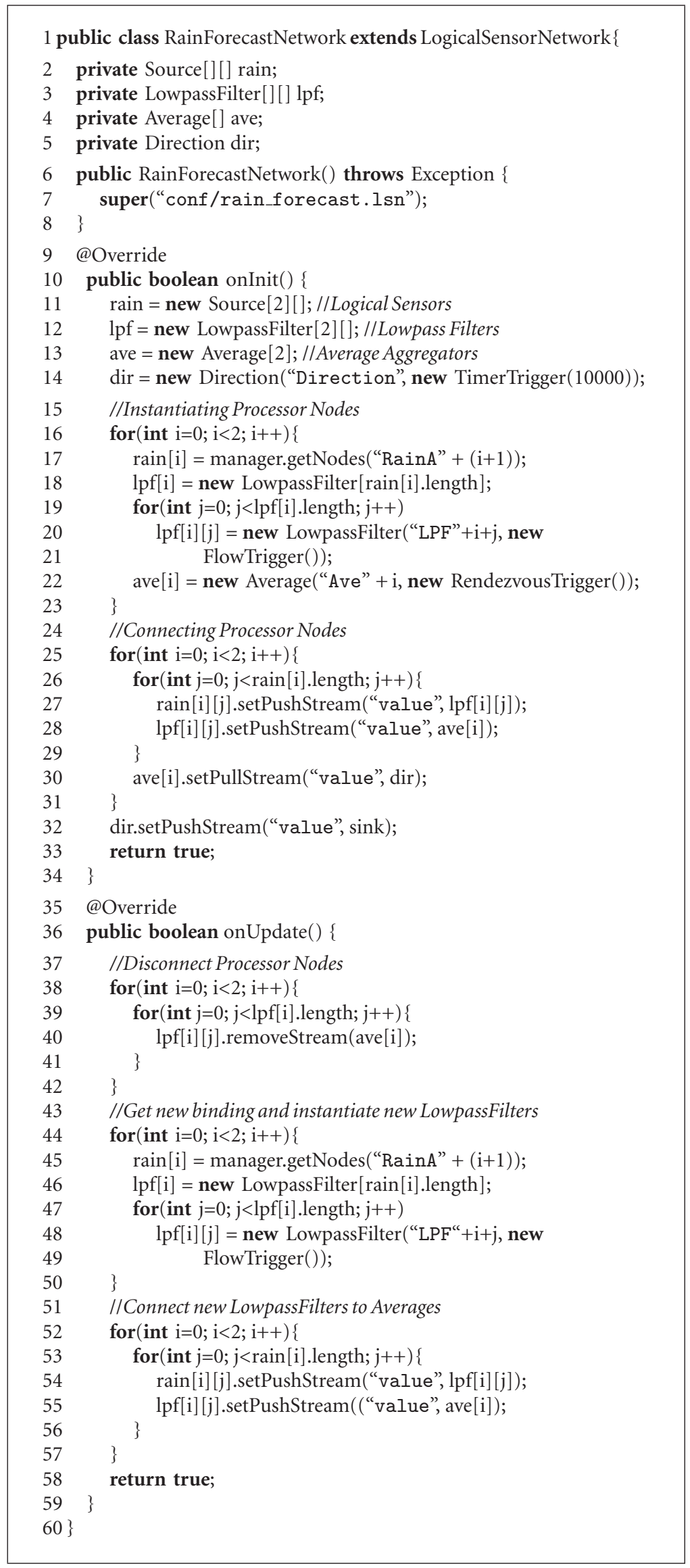

Listing 3: Source code for construction and update for logical sensor network in Java. 


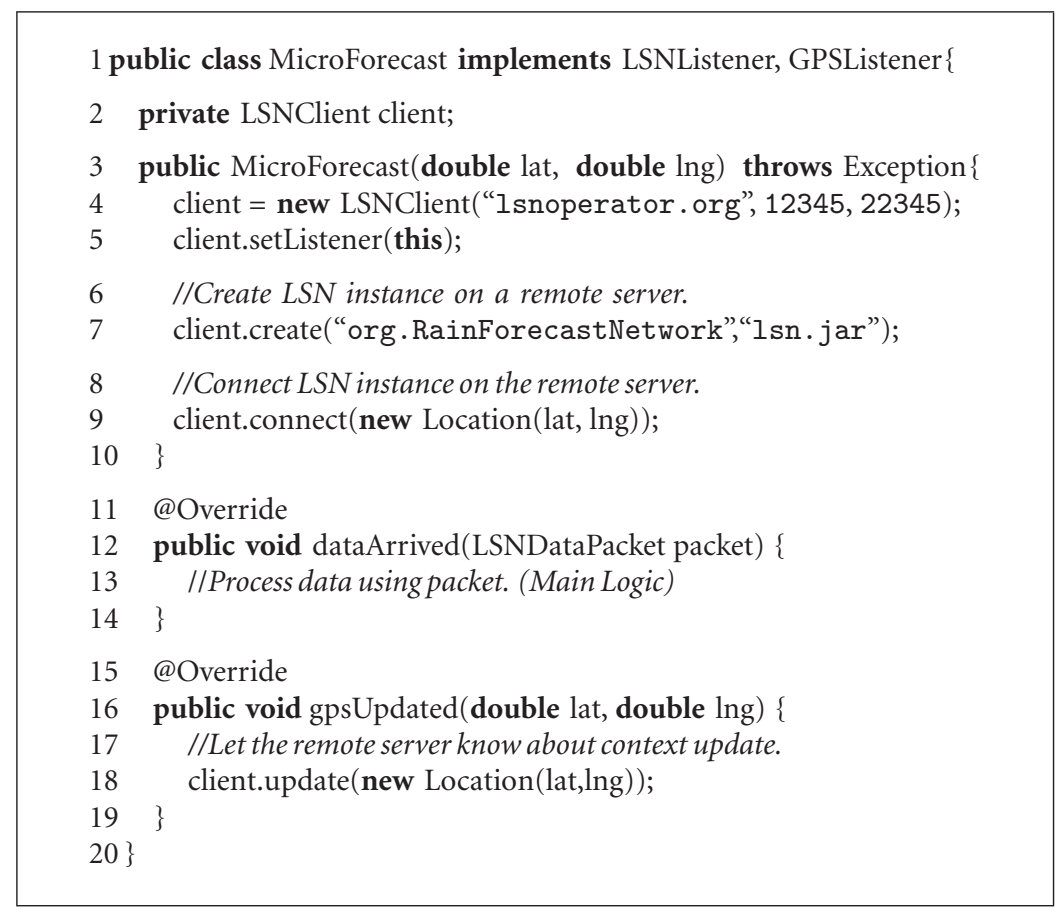

Listing 4: Source code for construction and update for logical sensor network in Java.

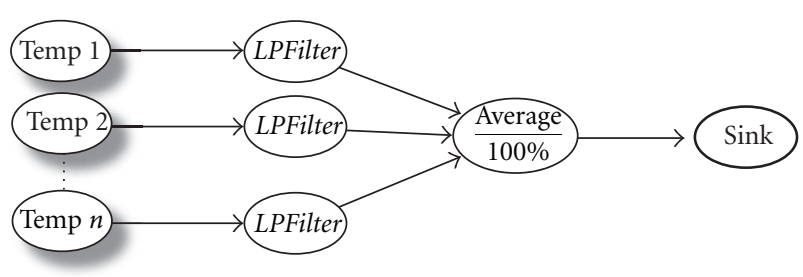

FIGURE 7: Logical Sensor Network used in the evaluation.

will be more significant. This evaluation verified that when processing sensor data from MDSN, applications should use LSN-Middle in the aspect of performance as well.

\section{Related Work}

Researches that focus on processing of sensor data can be seen in the field of macroprogramming. Titan [21], proposes a simple abstraction for sensor data processing for heterogeneous physical sensor network. Titan represents data processing by a data flow from sensors to recognition results. The abstraction of processing is represented as task, data, and connection. Task represents an elementary computational functions such as classifier and filters, and connection represents data flow from task. The entire process is expressed as directional graph in which tasks are connected by connections. The process execution is distributed among multiple sensor nodes. For the distributed computation, Titan has a special task for a communication to send sensor data to a different sensor node. However, from the aspect of the process abstraction, a communication among physical sensors

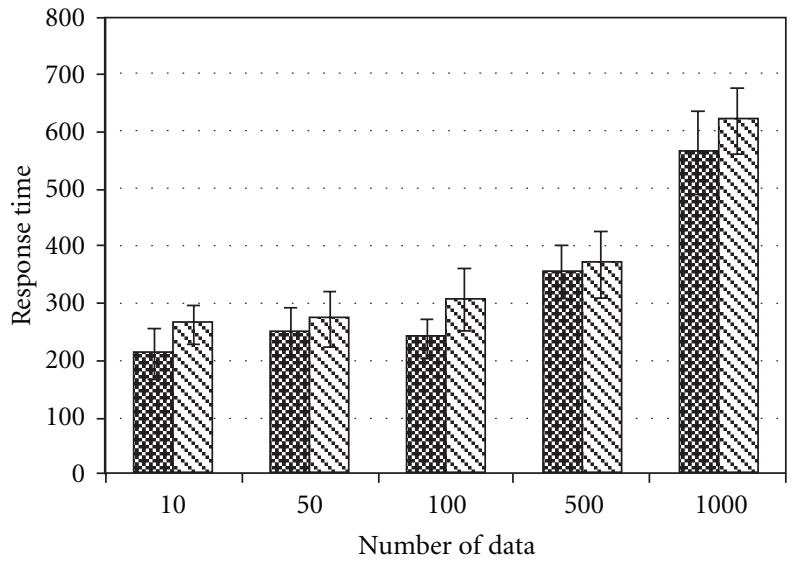

国 LSN mechanism

$\triangle$ Smartphone only

Figure 8: Performance evaluation of sensor data processing with and without LSN-Middle.

nodes should be transparent and not be shown in the process structure. If our middleware supports a distributed process execution, we will not change LSN structure, but add these settings as properties of a Node.

Abstract Task Graph (ATaG) [22] provides a sophisticated abstraction of sensor data processing. ATaG expresses sensor data processing with notion of task, data, and channel, which is called connection in Titan. Titan and ATaG have very close abstraction model, but in ATaG, task and channel have annotation, which can specify detailed behaviors. 
Annotations for task can specify the policy of task execution timing. Annotations for channel can specify the policy of data flow and selection of target tasks. ATaG uses a notion of task network with a more detailed abstraction in eventing and a communication aspect. The major difference is that ATaG has an element for data in its taskgraph. ATaG has to express data element as a part of the taskgraph structure since ATaG's assumption of task or process is too abstract that we can not recognize the output data from the name of the task or former tasks that are connected to it. On the other hand, the assumption of a process in LSN, which is called a task for ATaG, is a mathematical function that is small and general enough for people to know the output value from the name of the process. An implementation of process can be reused for other applications, by keeping it small and general.

\section{Conclusion}

In this research, we have claimed that the abstraction of sensor data processing proposed in the existing sensor network virtualization middleware over MDSN is not sufficient. Thus we have proposed a new abstraction called LSN to meet the requirements. An actual middleware and framework for sensor network virtualization was implemented and evaluated as well. Through the evaluation, we have found that despite of overhead that LSN-Middle requires, sensor data processing performance was $12.75 \%$ faster with LSN-Middle support in average compared to the result calculated in an application running on a smartphone. There are two future works for this research. One is to implement a graphical authoring tool for LSN to support application programmer to write and test LSN. And the other is to update the middleware for distributed execution of LSN since its structure is suitable for parallel processing. This can improve its performance and scalability.

\section{References}

[1] L. Luo, A. Kansal, S. Nath, and F. Zhao, "Sharing and exploring sensor streams over geocentric interfaces," in Proceedings of the 16th ACM SIGSPATIAL International Conference on Advances in Geographic Information Systems (GIS '08), pp. 250-259, November 2008.

[2] Y. Shiraishi, N. Thepvilojanapong, Y. Tamura et al., "TomuDB: multi-resolution queries in heterogeneous sensor networks through overlay network," in Proceedings of the 5th ACM International Conference on Embedded Networked Sensor Systems (SenSys '07), pp. 419-420, ACM, November 2007.

[3] H. Esaki and H. Sunahara, "Live E! project; Sensing the earth with internet weather stations," in Proceedings of the International Symposium on Applications and the Internet (SAINT '07), IEEE Computer Society, Hiroshima, Japan, January 2007.

[4] M. Isomura, T. Riedel, C. Decker, M. Beigl, and H. Horiuchi, Sharing Sensor Networks, IEEE, 2006.

[5] "Pachube:connecting environments, patching the planet," 2010, http://www.pachube.com/.

[6] N. Namatame, Y. Ding, T. Riedel, H. Tokuda, T. Miyaki, and M. Beigl, "A distributed resource management architecture for interconnecting web-of-things using uBox," in Proceedings of the 2nd International Workshop on the Web of Things (WoT '11), ACM, June 2011.
[7] A. Rowe, M. E. Berges, G. Bhatia et al., "Sensor andrew: largescale campus-wide sensing and actuation," IBM Journal of Research and Development, vol. 55, no. 1.2, pp. 6:1-6:14, 2011.

[8] V. Gupta, A. Poursohi, and P. Udupi, "Sensor.Network: an open data exchange for the web of things," in Proceedings of the 8th IEEE International Conference on Pervasive Computing and Communications Workshops (PERCOM '10), pp. 753-755, April 2010.

[9] V. Dyo, S. A. Ellwood, D. W. MacDonald et al., "Evolution and sustainability of a wildlife monitoring sensor network," in Proceedings of the 8th ACM International Conference on Embedded Networked Sensor Systems (SenSys '10), pp. 127-140, ACM, November 2010.

[10] K. Na, Y. Kim, and H. Cha, "Acoustic sensor network-based parking lot surveillance system," in Wireless Sensor Networks, U. Roedig and C. Sreenan, Eds., vol. 5432 of Lecture Notes in Computer Science, pp. 247-262, Springer, Berlin, Germany.

[11] G. Tolle, J. Polastre, R. Szewczyk et al., "A macroscope in the redwoods," in Proceedings of the 3rd International Conference on Embedded Networked Sensor Systems (SenSys '05), pp. 5163, ACM, 2005.

[12] J. Lu, T. Sookoor, V. Srinivasan et al., "The smart thermostat: using occupancy sensors to save energy in homes," in Proceedings of the 9th ACM International Conference on Embedded Networked Sensor Systems (SenSys '11), pp. 211-224, ACM, November 2011.

[13] M. Ito, Y. Katagiri, M. Ishikawa, and H. Tokuda, "Airy notes: an experiment of microclimate monitoring in shinjuku gyoen garden," in Proceedings of the 4th International Conference on Networked Sensing Systems (INSS '07), pp. 260-266, June 2007.

[14] S. Kabadayi, A. Pridgen, and C. Julien, "Virtual sensors: abstracting data from physical sensors," in Proceedings of the International Symposium on a World of Wireless, Mobile and Multimedia Networks (WoWMoM '06), pp. 587-592, IEEE Computer Society, June 2006.

[15] K. Aberer, M. Hauswirth, and A. Salehi, "A middleware for fast and flexible sensor network deployment," in Proceedings of the 32nd International Conference on Very Large Data Bases (VLDB '06), pp. 1119-1202, VLDB Endowment, 2006.

[16] K. Aberer, M. Hauswirth, and A. Salehi, "Infrastructure for data processing in large-scale interconnected sensor networks," in Proceedings of the 8th International Conference on Mobile Data Management (MDM '07), pp. 198-205, May 2007.

[17] S. Dawson-Haggerty, X. Jiang, G. Tolle, J. Ortiz, and D. Culler, "SMAP — a simple measurement and actuation profile for physical information," in Proceedings of the 8th ACM International Conference on Embedded Networked Sensor Systems (SenSys '10), pp. 197-210, ACM, November 2010.

[18] Sensor Modeling Language (Sensorml), 2012, http://www .opengeospatial.org/standards/sensorml.

[19] Y. Tian and E. Ekici, "Cross-layer collaborative In-network processing in multihop wireless sensor networks," IEEE Transactions on Mobile Computing, vol. 6, no. 3, pp. 297-310, 2007.

[20] K. Masaya, "D-jenga: a parallel distributed bayesian inference mechanism on wireless sensor nodes," in Proceedings of the International Conference on Networked Sensing Systems, 2006.

[21] C. Lombriser, D. Roggen, M. Stãd'ger, and G. Trãúster, “Titan: a tiny task network for dynamically reconfigurable heterogeneous sensor networks," in Kommunikation in Verteilten Systemen (KiVS), Informatik Aktuell, T. Braun, G. Carle, and B. Stiller, Eds., pp. 127-138, Springer, Berlin, Germany, 2007.

[22] A. Bakshi and V. K. Prasanna, "The abstract task graph: a methodology for architectureindependent programming of networked sensor systems," in Proceedings of the Workshop on End-to-end Sense-and-respond Systems (EESR '05), 2005. 

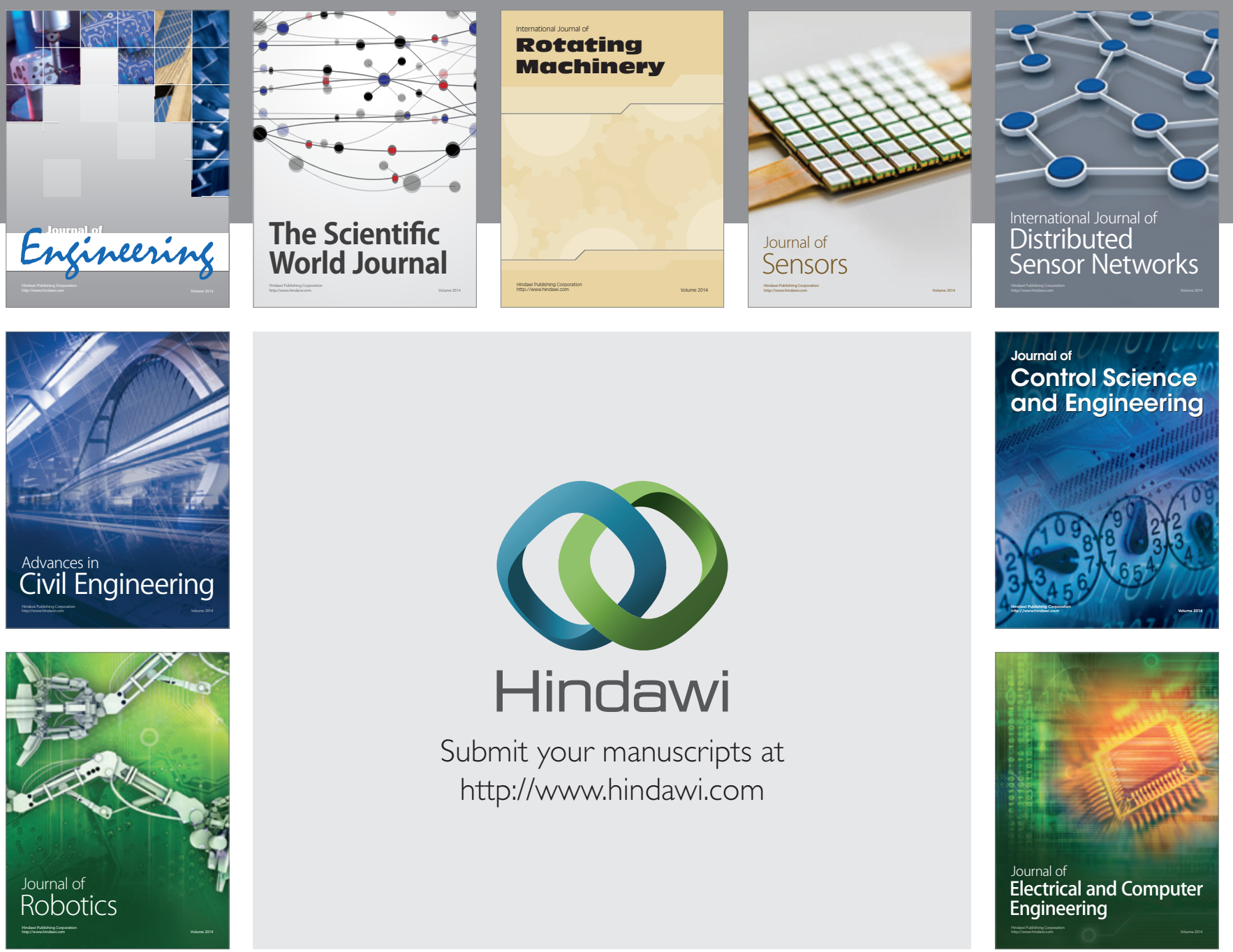

Submit your manuscripts at

http://www.hindawi.com
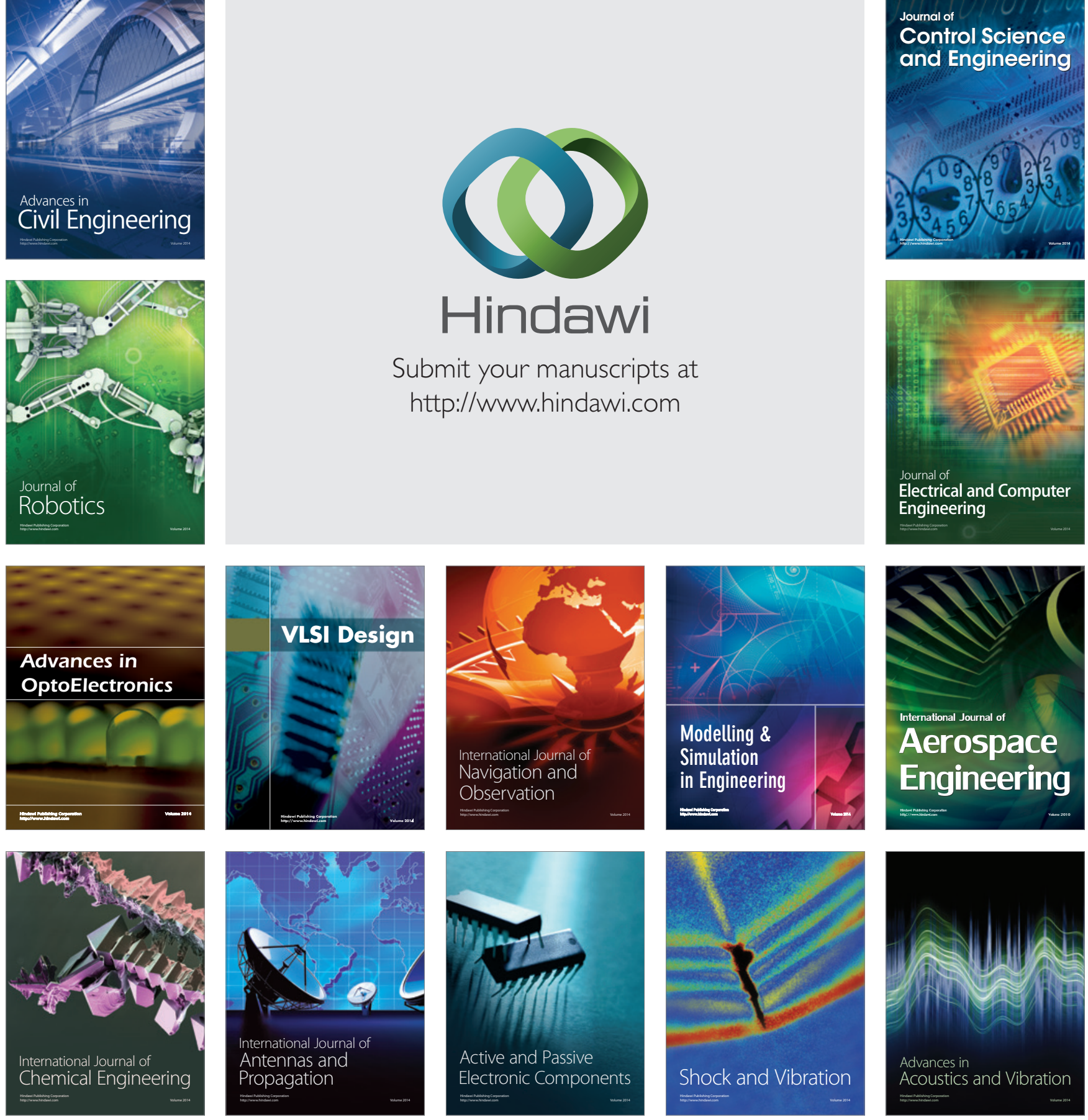